\title{
Biomechanical study of a semi-rigid stabilization system combined with transpedicular intracorporeal bone grafting for thoracolumbar burst fractures
}

xiaoyong zheng ( $\nabla$ zheng_yong_2000@163.com )

https://orcid.org/0000-0003-4225-8061

qingwen yu

8th medical center of Chinese PLA General Hospital

zhi zhang

8th medical center of Chinese PLA General Hospital

Research article

Keywords: Semi-rigid fixation, Non-fusion technique, Thoracolumbar, Burst fracture, Biomechanics

Posted Date: April 9th, 2020

DOI: https://doi.org/10.21203/rs.3.rs-20823/v1

License: (c) (i) This work is licensed under a Creative Commons Attribution 4.0 International License.

Read Full License 


\section{Abstract}

Background: For fresh thoracolumbar burst fracture, a new method which can not only promote the fracture healing, but also retain the movement segment, and restore the spinal movement function to the maximum extent is needed. The purpose of this study is to determine the performance of stabilization of a semi-rigid stabilization system combined with transpedicular intracorporeal bone grafting for thoracolumbar burst fractures.

Methods Six thoracolumbar cadaver spines were used for testing. A controlled L2 burst fracture was created. The L1-3 motions were determined.

Results In extension, flexion and lateral bending, the semi-rigid fixator stabilized the segment to a range of motion(ROM) and neutral zone(NZ) below the magnitude of the intact spine, but showed increased ROM and NZ of axial rotation $(P<0.05)$ compared with the intact spine.

Conclusions Restoration of stability with the semi-rigid dynamic system combined with transpedicular intracorporeal bone grafting is possible in flexion, extension, right and left lateral bending for thoracolumbar burst fracture but for axial rotation.

\section{Background}

Spinal canal decompression, short segment rigid fixation through pedicle, and

spinal fusion are the traditional surgical methods for thoracolumbar burst fracture, but they will inevitably lead to the loss of motor function of fixed segments, implant failure or other complications [1,2]. In theory, the anterior and middle columns of the injured vertebrae can be fully supported by bone grafting through the pedicle of the injured vertebrae without spinal segment fusion $[3,4]$, and the complications such as motor function loss caused by intervertebral fusion can also be avoided [5]. However, its combination with rigid internal fixation system violates its original intention of non-fusion. In previous studies, we found that if there is disc injury before operation, the rate of disc degeneration after operation is as high as $42.86 \%$, while the rate of disc degeneration without injury before operation is $16.67 \%$. This can be used to explain that the problem of loss of correction angle can not be avoided by intravertebral bone grafting. In the clinical treatment, we need a new method that can not only promote the fracture healing, but also retain the segment motion, and restore the spinal movement function to the maximum extent. In this study, we used a semi-rigid internal fixation system. By establishing a cadaver model of thoracolumbar burst fracture, we observed the effect of semi-rigid internal fixation combined with transpedicular bone graft on thoracolumbar burst fracture.

\section{Methods}

1. Semi-rigid internal fixation system (Figure 1-2): The semi-rigid fixation system is 
made of titanium alloy for surgical implants, which has been used in clinic. It is composed of semi-rigid connecting rod, posterior opening "U" pedicle screw. The semi-rigid connecting rod includes titanium alloy rod, cable, disc spring, pin, etc[6]. Titanium cable and disc spring provide a certain degree of activity and elasticity, so as to achieve the purpose of semi-rigid fixation. (Patent No.: ZL 20092 0175651.9). The buckling range of the connecting rod is $0-10^{\circ}$, and the rotation range is $0-5^{\circ}$. Model and specification of semi-rigid connecting rod includes $6 \mathrm{~mm} \times 90 \mathrm{~mm}$ and $6 \mathrm{~mm} \times 120 \mathrm{~mm}$.

2. Rigid internal fixation system:

Including pedicle screws and rigid connecting rods, the diameter of connecting rod is $6 \mathrm{~mm}$, made of medical titanium alloy. Provided by Beijing Fule Technology Development Co., Ltd.

3. Test equipment:

1) MTS 858 material testing system (MTS, USA).

2) Spinal 3D motion analysis system:

The digital motion capture system is composed of six digital motion capture lenses around the site (Model EAGLE4, made by Motion Analysis Company, with pixel value of 4 million, maximum acquisition frequency of 2000 frames per second, theoretical accuracy of $0.02 \mathrm{~mm}$, rotation angle measurement of 0.02 degree). The data are directly input into the computer, and the software automatically analyzes and calculates the angle change of three-dimensional spine movement.

\section{Test specimen:}

Six fresh cadaveric thoracolumbar specimens (T12-L3, 28-43 years old, average 31.7 years old, from the Department of anatomy, Southern Medical University, CHINA) were selected. Which exclude spinal trauma, variation, tumor and hyperosteogeny. The specimens were wrapped with multi-layer film and stored in

$-20^{\circ}$ freezer. Referring to the literature, we successfully made a burst fracture model of L2 vertebral body by means of compression after pre injury. After the L2 vertebral body was pre drilled, the adjacent vertebral body and intervertebral disc were embedded in high-strength gypsum, and the specimen vertebral body was impacted by the free drop hammer method $(15 \mathrm{~kg}, 1.1 \mathrm{~m}$ high). As a result, the L2 vertebral body fracture, the bone block entering the vertebral canal, the small joint relaxation, the upper and lower endplates of all specimens were fractured, which belonged to Denis A type fracture. All specimens were successfully modeled at one time (Fig. 2).

Each specimen was tested in four consecutive states: complete state, fracture state, rigid fixation system and semi-rigid internal fixation system. Two hours before the test, the frozen specimens were taken out, thawed naturally and completely at $25^{\circ} \mathrm{C}$ room temperature, then covered with wet saline gauze to keep the specimens moist. Drill the Kirschner wire into the L1 and L3 vertebrae and install the marked points. 
The specimen was fixed on a three-dimensional spinal motion testing machine with a loading moment of $8 \mathrm{~N} . \mathrm{m}$. Load from $1 \mathrm{~N} . \mathrm{m}$ to $8 \mathrm{~N} . \mathrm{m}$ step by step, the loading rate is $0.2 \mathrm{~mm} / \mathrm{sec}$, three times of loading in all directions, the first two times to eliminate the influence of creep. At the third time, test the angle displacement of specimen forward bending, backward extension, left bending, right bending, left turning and right turning, and take this as the reference data. Then the above specimens were made into thoracolumbar burst fracture model (L2). Six three-dimensional motion indexes of spine were measured. For each model, the end plate fracture block was fixed through the bilateral pedicle drilling. The holes of the arch root of the vertebra were enlarged with series guide cones of different diameters (Diameters up to $7 \mathrm{~mm}$ ). The depth of the guide cones should reach to the anterior column of the vertebra. The guide cones were inserted into the bone grafting funnel, and the bone particles were filled and implanted into the vertebra. The guide cones were used to compact the holes (Fig. 3).

Six three-dimensional motion indexes of spine were measured after transpedicular bone grafting. Pedicle screws were implanted in L1 and L3 bilateral pedicle, and six three-dimensional motion indexes of spine were measured by using semi-rigid connecting rod (Figure 4). Six three-dimensional motion indexes of spine were tested.

7区 Statistical analysis

SPSS16.0 software is used for statistics and analysis. Random block analysis of variance was used to compare ROM and NZ in different states. The significance level was 0.05 .

\section{Results}

The results showed that the ROM and NZ in all directions of the bone grafting group had no significant difference compared with the fractured group $(P>0.05)$ (Table 1 and table 2$)$, and it was still far greater than the intact group $(P<0.01)$, suggesting that the bone grafting in the injured vertebra was not enough to provide enough stability. After rigid internal fixation, ROM and NZ in the flexion, extension and lateral flexion direction of the specimen decreased significantly, which was significantly different from that in the fractured group and intact group $(P<0.01)$, but ROM and NZ in the rotation direction was almost the same as that in the intact group, with no significant difference $(P>0.05)$. Compared with the fractured group, ROM and NZ of the semi-rigid group in flexion, extension and lateral flexion direction decreased significantly $(P<0.01)$, while ROM and NZ in rotation direction decreased significantly $(P<0.01)$, but it was significantly greater than that in intact state, and the difference was significant $(P<0.01)$.

\section{Conclusions}

Due to the change of stress, the hydration capacity of extracellular matrix of intervertebral disc decreases. Abnormal spinal load can reduce the hydration capacity and protein content of intervertebral disc. This leads to disc degeneration. As a result, the height of intervertebral space is reduced, the pressure of small joints is increased, and the range of motion between spinal segments is changed. Rigid internal fixation 
has a direct effect on the internal pressure of intervertebral disc. It has been proved that the overload or under load of the intervertebral disc is harmful to the disc. Rigid internal fixation also has a direct effect on the metabolism of intervertebral disc. The brake of the disc stops the pressure change in the disc, and the fluid flow inside and outside the disc stops, which is driven by the change of pressure circulation. Kurakawa et al. ${ }^{[7]}$ showed that the material exchange in and out of the fixed segment of the disc became slow, while that in the adjacent segment increased, which also led to the degeneration of the disc. Therefore, in order to avoid the degeneration of fixed segment disc and promote the repair of damaged disc, it is necessary to improve the existing fixation methods to create a suitable mechanical environment, delay the degeneration of disc and reduce the incidence of complications such as implant failure.

In the treatment of thoracolumbar burst fracture, transpedicular bone grafting is considered as one of the measures to solve the failure of internal fixation because it can provide extra support for the anterior and middle columns of the vertebral body. But the clinical report is controversial. The main reason is that transpedicular bone grafting can not avoid the degeneration of the injured disc. In order to solve this problem, we intend to provide a certain amount of stress to the fracture site and the injured disc, the purpose is to promote the repair of the injured disc and the healing of the fracture. In this experiment, we successfully made a fresh cadaver model of $L 2$ vertebral burst fracture by using the method of free drop hammer after specimen pre injury. In morphology, we can find that L2 vertebral fracture, bone mass entering into vertebral canal, small joint relaxation, all the upper and lower endplates of the specimens are fracture, which belongs to Denis A type fracture. The biomechanical test results showed that the stability of the spine was significantly lower than that of the intact spine, and the difference was significant, indicating that the model was successful.

The results showed that transpedicular bone grafting alone is not enough to provide enough stability. Semi-rigid internal fixation combined with transpedicular bone grafting can maintain the stability of thoracolumbar burst fracture in flexion, extension and lateral flexion, but not enough in rotation direction. In order to improve the stability of the internal fixator, we all added the transverse connector. The results showed that the ROM of the flexion, extension and lateral flexion direction of the specimen after the rigid fixation and the use of the transverse connector was significantly reduced, which was significantly different from the fracture state and the intact state, but the ROM of the rotation direction was basically similar to the intact state, and the difference was not significant $(P>0.05)$, suggesting that the transverse connector was effective for the fixation of the internal fixator. The effect on improving the rotation stability is limited. In addition, the semi-rigid internal fixation system can provide a certain degree of rotation, so its combination with transpedicular bone graft is not enough to provide enough stability in the direction of rotation for thoracolumbar burst fracture.

There are many shortages in this experiment. For example, the factors of individual differences between specimens, the factors of paravertebral muscles and so on. In addition, in normal human physiological state, the healing process after fracture and the effect on the internal fixation stress are not included. Moreover, due to the limitation of objective conditions, this experiment has not been tested for shear resistance, which is also one of the problems that need to be paid attention to in the further study. We will 
improve the semi-rigid connecting rod, increase its stability, provide continuous stress to the fracture end, and reduce the shear force and rotation stress as much as possible, so as to provide a new method for clinical treatment of thoracolumbar burst fracture.

\section{Declarations}

\section{Ethics approval and consent to participate}

The study was approved by the ethics committee of the eighth medical center of PLA General Hospital

\section{Consent for publication}

Not applicable.

\section{Availability of data and materials}

The datasets used and/or analysed during the current study are available from the corresponding author on reasonable request.

\section{Competing interests}

The authors declare that they have no competing interests.

\section{Funding}

The study was supported by the nursery fund of PLA General Hospital

\section{Authors' contributions}

Zheng Xiaoyong carried out the experiment, analyzed the data and wrote the manuscript. Yu Qingwen participated in the experiment and data analysis. Zhang zhi participated in the experiment and data analysis. All authors read and approved the final manuscript.

\section{Acknowledgements}

Not applicable.

\section{Abbreviations}

ROM range of motion

NZ neutral zone

\section{References}


1. Dong SH, Chen HN, Tian JW,et al. Effects of minimally invasive percutaneous and trans-spatium intermuscular short-segment pedicle instrumentation on thoracolumbar mono-segmental vertebral fractures without neurological compromise. Orthop Traumatol Surg Res. 2013; 99(4): 405-411.

2. Tofuku K, Koga $\mathrm{H}$, ljiri K,et al. Combined posterior and delayed staged mini-open anterior shortsegment fusion for thoracolumbar burst fractures. J Spinal Disord Tech. 2012; 25(1): 38-46.

3. Liao JC, Fan KF, Keorochana G, et al. Transpedicular grafting after short-segment pedicle instrumentation for thoracolumbar burst fracture:calcium sulfate cement versus autogenous iliac bone graft. Spine. 2010; 35(15):1482ه1488.

4. Li DP, Yang HL, Huang YH, et al. Transpedicular intracorporeal grafting for patients with thoracolumbar burst fractures. Saudi Med J. 2014; 35(1): 50-55.

5. Toyone T, Ozawa T, Inada K, et al. Short-segment fixation without fusion for thoracolumbar burst fractures with neurological deficit can preserve thoracolumbar motion without resulting in posttraumatic disc degeneration: a 10-year follow-up study. Spine (Phila Pa 1976). 2013; 38(17): 14821490.

6. Zheng Xiaoyong, Zhao Dongsheng, Ma Yuanzheng, et al. Prospective design and stability of a new dynamic stabilization system for lumbar spine. Orthopaedic Biomechanics Materials and Clinical Study. 2015; 12(4): 75-80.

7. Kurakawa $\mathrm{T}$, Kakutani $\mathrm{K}$, Morita $\mathrm{Y}$, et al. Functional impact of integrin a5 $\beta 1$ on the homeostasis of intervertebral discs: a study of mechanotransduction pathways using a novel dynamic loading organ culture system. Spine J. 2015; 15(3): 417-426.

\section{Tables}

Table 1 ROM of L1-3 segment in various states (degrees, $x \pm s$ )

\begin{tabular}{lcccccc}
\hline & Intact & Fractured & Bone grafting & semi-rigid & rigid & \\
\hline Flexion/extension & $22.68 \pm 0.35$ & $44.49 \pm 0.74$ & $43.36 \pm 1.25$ & $6.38 \pm 1.84$ & $4.26 \pm 0.94$ \\
Lateral flexion & $14.66 \pm 0.18$ & $35.12 \pm 3.96$ & $36.97 \pm 1.03$ & $5.65 \pm 0.79$ & $2.26 \pm 0.16$ \\
Rotation & $10.77 \pm 1.16$ & $26.13 \pm 5.52$ & $25.29 \pm 4.71$ & $13.18 \pm 0.28$ & $10.08 \pm 0.38$ \\
\hline
\end{tabular}

Table 2 Neutral range of L1-3 segment in various states (degrees, $\mathbf{x} \pm \mathbf{s}$ )

\begin{tabular}{lccccccc}
\hline & Intact & Fractured & Bone grafting & semi-rigid & rigid \\
\hline Flexion/extension & $7.81 \pm 0.54$ & $14.46 \pm 0.02$ & $14.29 \pm 3.88$ & $4.52 \pm 0.88$ & $2.79 \pm 0.39$ \\
Lateral flexion & $5.65 \pm 1.24$ & $13.71 \pm 7.16$ & $12.17 \pm 7.04$ & $4.66 \pm 0.02$ & $1.38 \pm 0.07$ \\
Rotation & $2.97 \pm 0.88$ & $8.65 \pm 2.51$ & $7.85 \pm 0.25$ & $4.92 \pm 0.36$ & $2.69 \pm 0.27$ \\
\hline
\end{tabular}

\section{Figures}




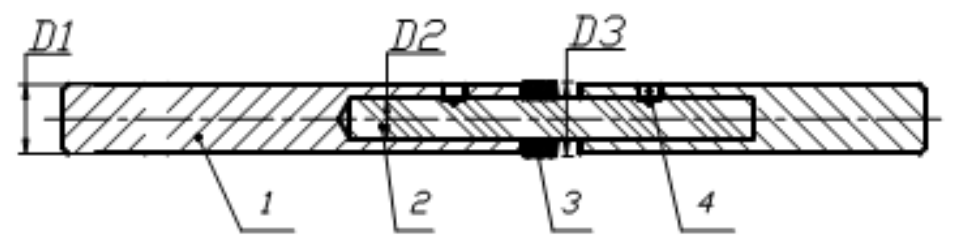

1. Fixed rod 2. Titanium alloy cable 3. Disc spring 4. Pin

$\mathrm{D} 1=6 \mathrm{~mm}, \mathrm{D} 2=3.2 \mathrm{~mm}, \mathrm{D} 3=6.4 \mathrm{~mm}$

\section{Figure 1}

Structure diagram of semi-rigid connecting rod

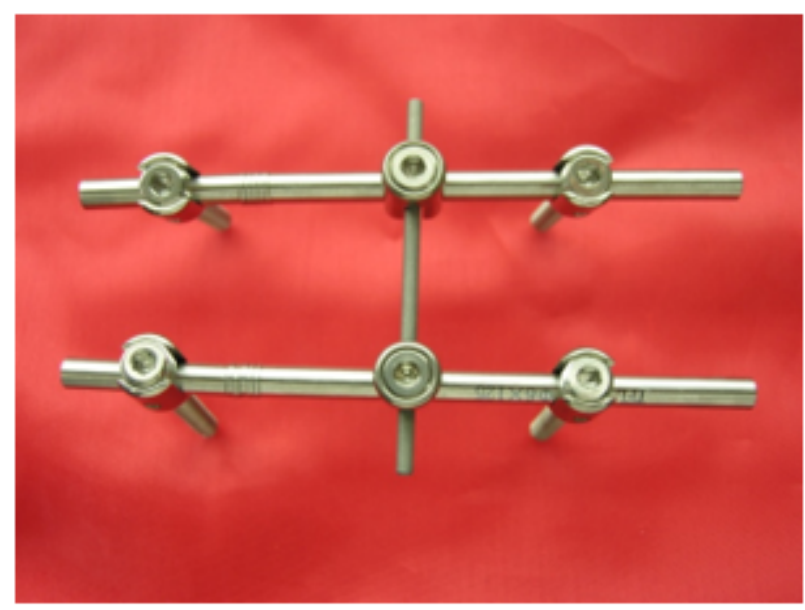

Figure 2

Semi-rigid internal fixation system

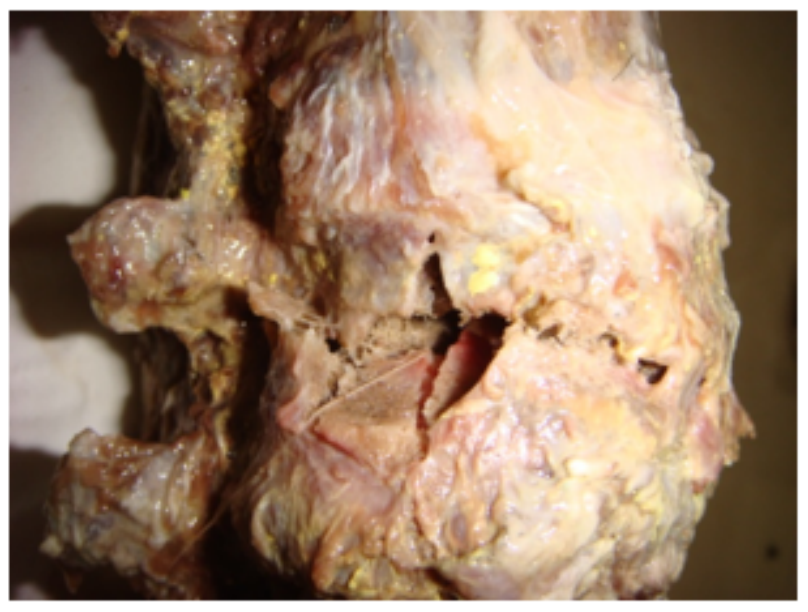

Figure 3

Model of $L 2$ vertebral burst fracture 


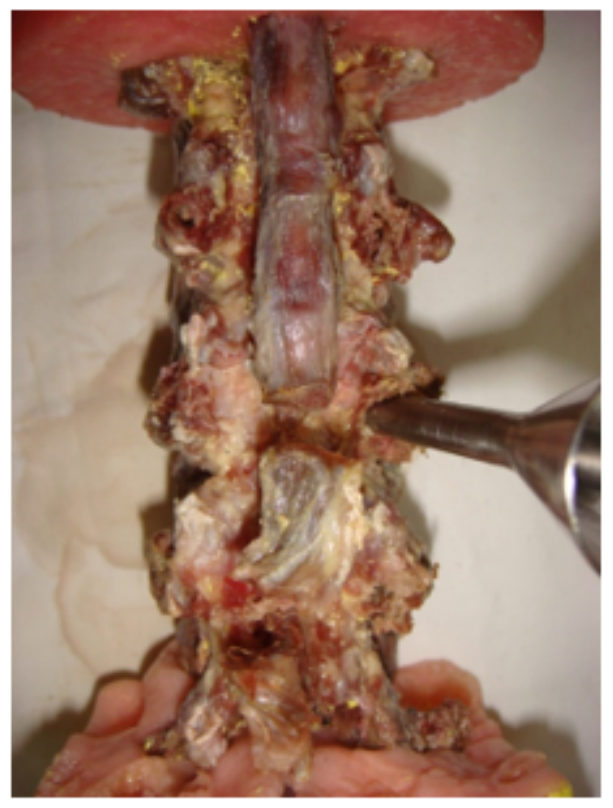

\section{Figure 4}

Bone graft through L2 pedicle

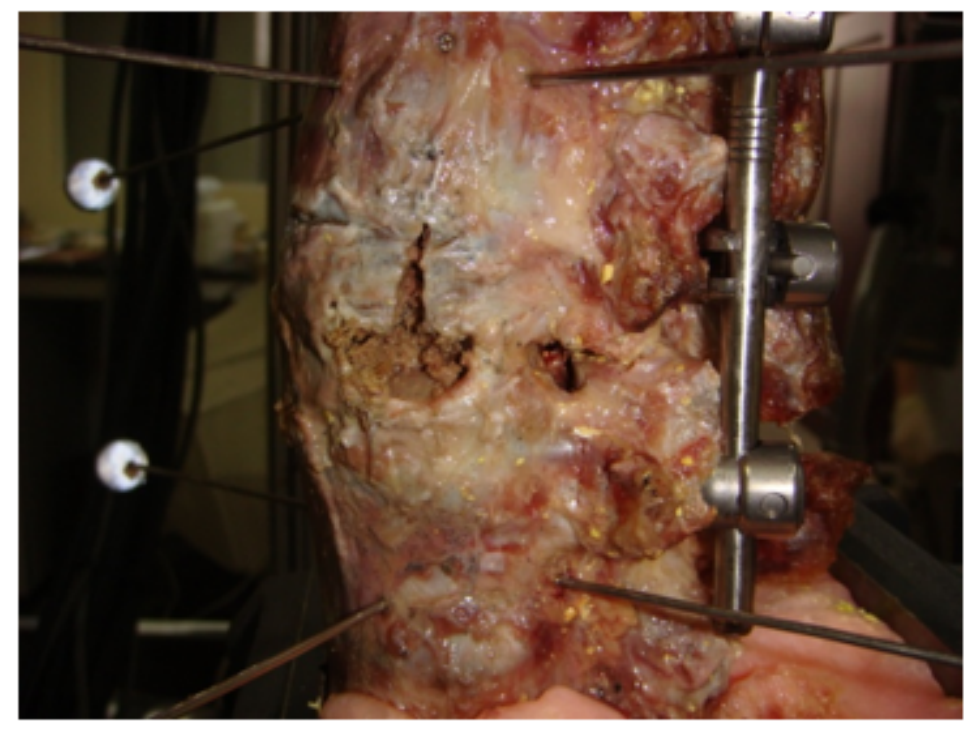

Figure 5

Semi-rigid internal fixation after bone grafting 